\title{
Ed Shaya
}

Department of Physics, U. of Maryland

As a followup to Bel Campbell's presentation of Planetary and Galactic images, I am presenting Extragalactic images recently obtained by the WF/PC Instrument Definition Team (IDT) using the Wide Field/Planetary Camera onboard the HST. I have many exciting results and little time/space here, therefore, the descriptions will be quite brief and will only highlight the most interesting new science or capablities of the instrument. Many of the images have been published elsewhere and I refer to them whenever possible.

M15 - (Lauer and WF/PC IDT 1991a) - A PC image obtained by four 800 s integrations through filter $\mathrm{F} 336 \mathrm{~W}$ of the bright globular cluster M15 resolved all the red giant stars at the core of M15 for the first time. The light of the giant stars were subtracted using DAOPHOT algorithms for point spread function fitting. The underlying light distribution from main sequence stars flattens off interior to $\mathrm{r}=2.2$ arcsec $=0.13 \mathrm{pc}$. M15 has no cusp at the center, but the distribution of a small number of giant stars would mislead one into measuring a cusp if they are not subtracted (Fig. 1).

NGC 7457 - (Lauer and WF/PC IDT 1991b) - This S0 galaxy at 12.5 Mpc distance has a light curve that follows $\mu(r) \propto r^{-1,0}$ in its core down to the resolution limit of 0.05 arcsec or $3.2 \mathrm{pc}$. The luminosity density within a resolution element of the core reaches $3 \times 10^{4} L_{\odot} p c^{-3}$. Only M32 is known to have a denser stellar core than this (Fig. 2).

NGC 1068 - (Lynds and WF/PC IDT 1991) - This Seyfert Galaxy has been intensively studied for more than 20 years. The one 180 s exposure through the filter F $547 \mathrm{M}$ marginally resolves the core to be of size 0.15 arcsec or $11 \mathrm{pc}$. The broad line region is therefore seen indirectly through scattering through a cloud in the vicinity. Deconvolution of the image reveals that the resolved core is slightly off-center to an oblong bright region with dimensions of $3.5 \times 1.7 \operatorname{arcsec}$ or $255 \times 124 \mathrm{pc}$ (Fig. 3). This bright region does not appear in the narrow band images (Evans $e t$ al.) and therefore we conclude it is composed of stars.

M87 - (Lauer and WF/PC IDT 1992) - Two 700s PC exposures through the F785LP filter of this giant elliptical in the Virgo Cluster reveal a power law starlight profile of the form $\mu(r) \propto r^{-1 / 1}$ plus a central nonthermal point source (Fig. 4). The cuspy light distribution is consistent with the model of Young et al. (1978) in which there is a $2.6 \times 10^{9} \mathrm{M} \odot$ black hole at the center. The image also reveals jet structure details to within one resolution element of the core.

NGC 1275 - (Holtzman and WF/PC IDT 1992) - PC observations in filters F555W, F702W, $\mathrm{F} 664 \mathrm{~N}$, and $\mathrm{F} 673 \mathrm{~N}$ were obtained of this active galaxy at the center of the Perseus Cluster. Its 
nucleus is surrounded by approximately 50 blue and bright point-like sources and several diffuse blue sources (Fig. 5). Only one of these shows strong $H \alpha$ emission lines. That object corresponds to the one discovered by Shields and Filipenko (1990). We hypothesize from their colors and luminosities that these sources are young (less than $2 \times 10^{8} \mathrm{yr}$ ) globular clusters. They range in luminosity up to 100 times the brightest globular clusters in the Milky Way System. These clusters may be forming out of the cooling flow which has been inferred from X-ray observations. Their formation may have been triggered by a recent merger with a neighboring galaxy. An indication that a merger has occurred recently is the existence of shell structure near the nucleus seen in the $\mathrm{WF} / \mathrm{PC}$ images.

The remainder of this talk is a summary of gravitational lenses observed with WF/PC by the IDT. In general, we should be able to measure relative positions of quasar images to an accuracy of about 1 milliarcsec and relative brightnesses to 2 percent. But, most importantly, the HST WF/PC can resolve the quasar images and lensing galaxy with .1 arcsec resolution.

Q0957+561 - the "Double Quasar" - The F555W and F785LP images (Fig. 6) easily resolve the lensing galaxy from the brighter quasar image. Point spread function subtraction of the brighter image allows one to measure the radial light distribution of the main lensing galaxy.

PG1115+080 - was the "Triple Quasar" - Four distinct quasar images plus the lensing galaxy are clearly seen (Fig. 7). The two outer images are near a caustic and therefore their stellar-like appearances strongly constrain the size of any possible jet around the quasar.

Q2345+007 - Only two quasar images were present at the time of the observation. Earlier reports that one of the images is split into two is not confirmed.

Q2237+0305 - the "Einstein Cross" - The galaxy nucleus and four quasar images are easily resolved. As in the FOC image, no sign of the predicted fifth image nearest to the nucleus is present.

\section{CONCLUSION}

Bel Campbell and I have presented most, but not all, of the important new science results thus far from HST WF/PC for the IDT. This is a small fraction of what is planned for the next few years. With nearly every exposure taken, significant new astronomical information is obtained. I agree with the comments of other speakers here today, that the loss due to spherical aberration of the primary mirror is mostly in signal-to-noise, particularly so in dealing with faint objects near to bright objects, but the resolving power in most situations is nearly equal to pre-launch specifications. This allows users to obtain unique and useful scientific data on bright objects. In conclusion, the $\mathrm{HST} \mathrm{WF} / \mathrm{PC}$ is a viable working instrument.

\section{REFERENCES}

Evans, I. N., Ford, H. C., Kinney, A. L., Antonucci, R. R. J., Armus, L., and Caganoff, C., 1991, ApJ Letters, 369, L27.

Holtzman, J. A. and WF/PC IDT 1992. Submitted to ApJ.

Lauer, T. R., and WF/PC IDT, 1991a, ApJ Letters, 369, L41.

Lauer, T. R., and WF/PC IDT, 1991b, ApJ Letters, 369, L45.

Lauer, T. R., and WF/PC IDT 1992. Submitted to $A J$.

Lynds R., and WF/PC IDT 1991, ApJ Letters, 369, L31.

Shields, J. C., and Filipenko, A. 1990, $A p J, 353, \mathrm{~L} 7$.

Young, P. J., Westphal, J. A., Kristian, J., Wilson, C. P., and Landauer, F. P., 1978, A pJ, 221, 721.

The WF/PC IDT consists of W. A. Baum, D. G. Currie, S. P. Ewald, S. M. Faber, E. J. Groth, J. J. Hester, J. A. Holtzman, J. Kristian, R. M. Light, C. R. Lynds, E. J. O'Neil, Jr., D. P. Schneider, E. J. Shaya, and J. A. Westphal. 


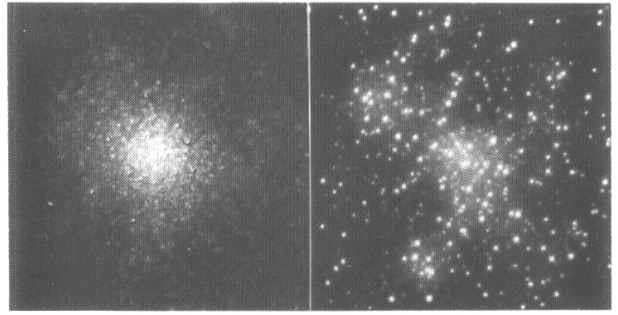

Fig. 1. Centra1 17" of M15 a) the 1ight distribution of underly1ng main seguence staxs. b) DAOPHOT generazed 1mage of giant stars with ST point spread functions.

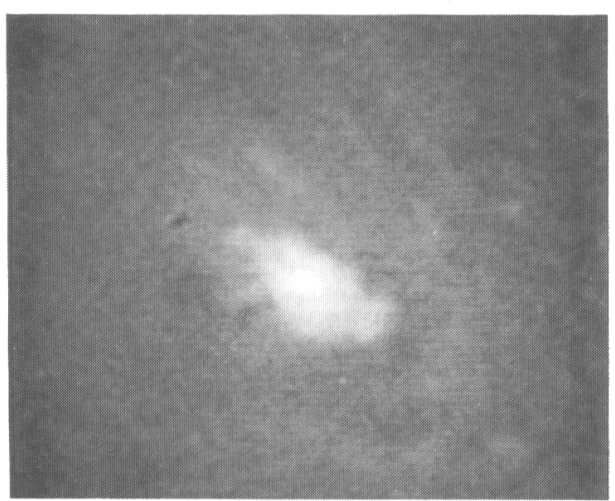

Fig. 3. Central region of NGC 1068 after 85 iterations of Lucy deconvolution. The central spike is marginally resolved and is surrounded by irregular shaped star cluster.
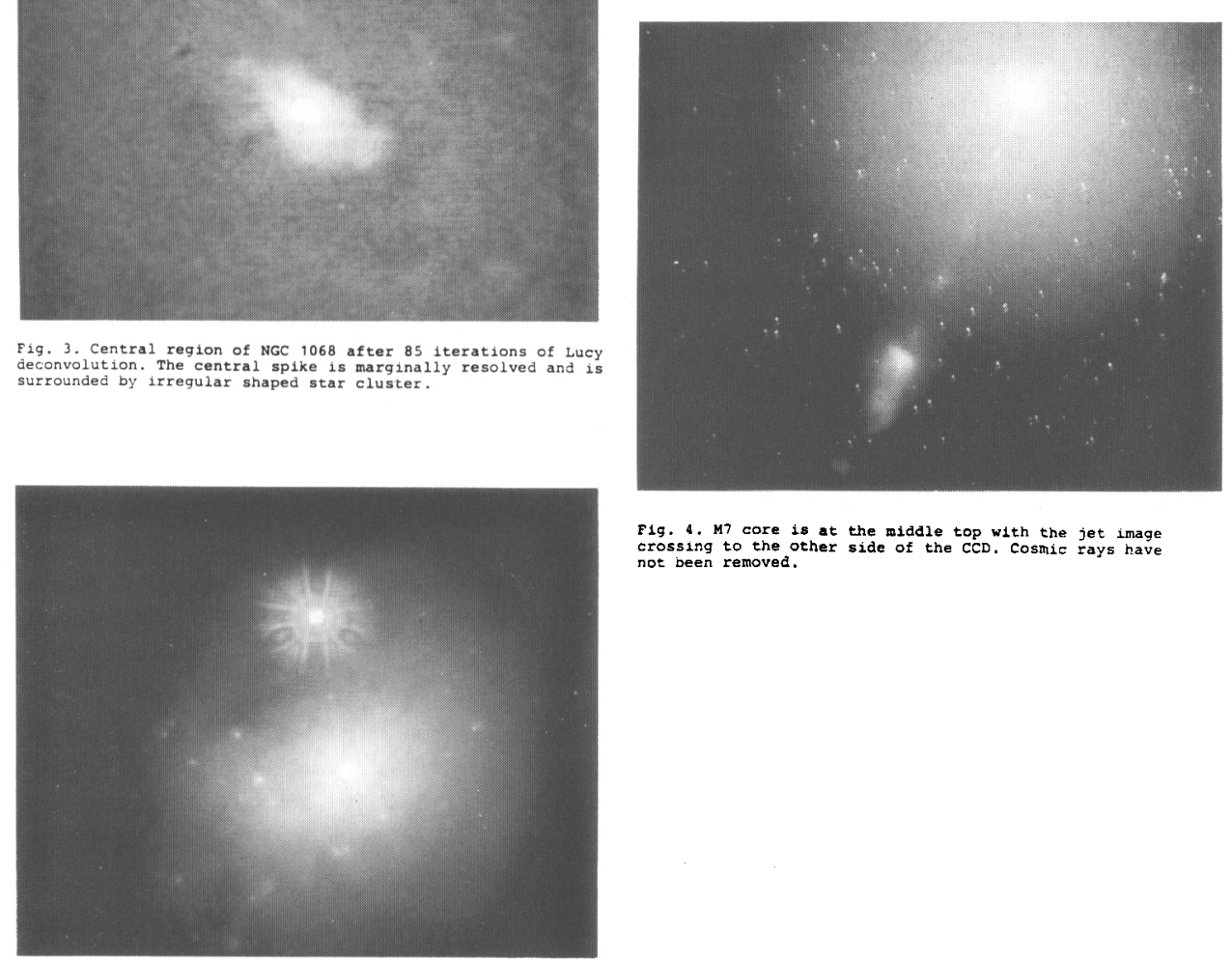

F1g. 4. M7 core is at the middle top with the jet image crossing to the other side of the $\mathrm{CCD}$. cosmie rays hav
not been removed.

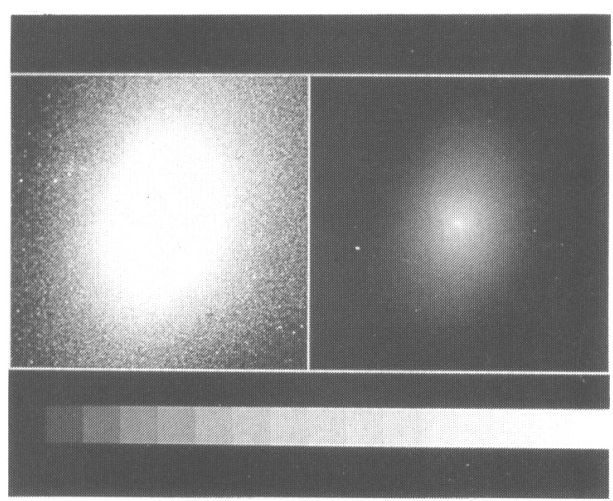

Fig. 2. Central 17" of NGC 7457 with two different stretches. The compact core is evident on the right.

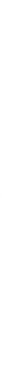

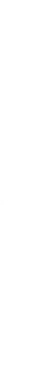

Fig. 5. Central ragion of NGC 1275. Top of center is an F5 star, miadle is nucleus surrounded by many unresolved bright blue sources. 


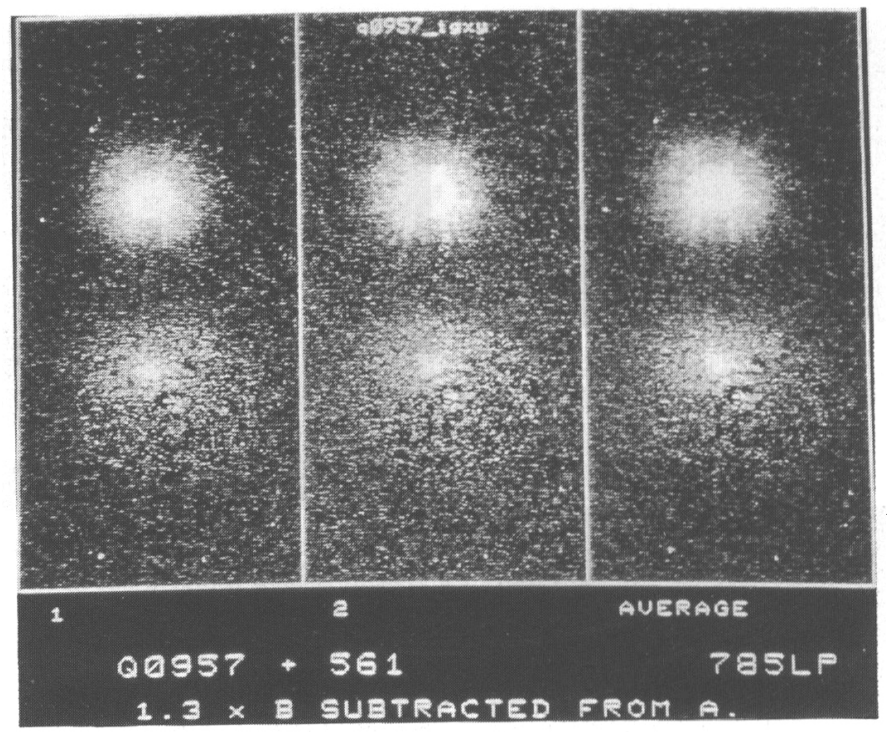

Pig. 6. Double Quasar. Bottom quasar image has been subtracted of using scaled up version of top quasar image. Bottom image is lensing galaxy $1^{\text {H }}$ from subtracted quasar image. Subtraction is not perfect because WF/PC PSF is spatially variable due to spherical aberration.

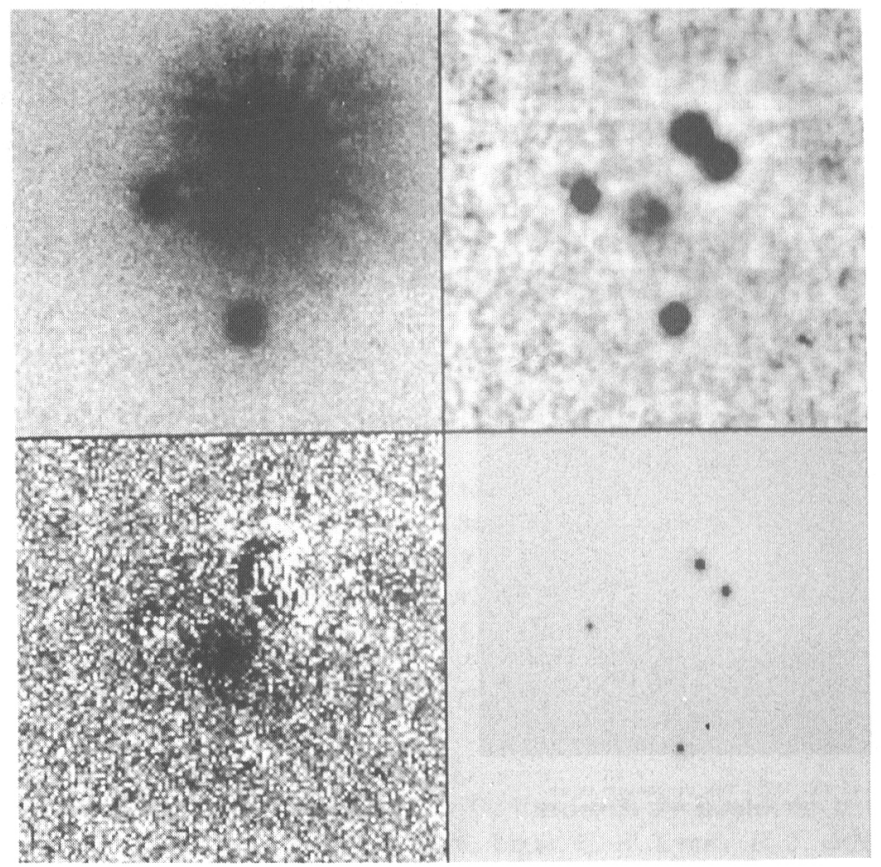

Fig. 7. PG1115+080. Top left) raw image; Top right) Lucy decor volved image shows 4 guasar images with lensing galaxy in the middle; Bottom right) Display stretch to show core. Images are separated by many ST resolution elements; Bottom left) Lensing galaxy after quasar images are subtracted. 